CELL BIOLOGY OF THE NEURON

\section{Directing neurofilament traffic}

Neurofilaments are components of the neuronal cytoskeleton that are important both for the maturation of axons and the maintenance of axonal integrity. The individual subunits that make up the neurofilaments are transported from the cell body to the axon, where they bundle together to form filaments. So how does the neuron ensure that the neurofilaments are assembled in the correct compartment of the cell? As Shea and colleagues report in the Journal of Cell Science, phosphorylation, and in particular the activity of the serine/threonine kinase Cdk5 (cyclin-dependent protein kinase 5), seems to be the key.

The authors investigated the effects of enhancing or inhibiting the activity of Cdk5 in cultured chick dorsal root ganglion neurons. They found that if they overexpressed
Cdk5 along with its activator $\mathrm{p} 35-\mathrm{a}$ manipulation that leads to increased phosphorylation of the carboxyl termini of neurofilament subunits - the neurofilaments aggregated to form thick bundles within the cell body instead of being transported into the axon. By contrast, when they treated the cells with the Cdk5 inhibitor roscovitine, the transport of neurofilament subunits into the axon was enhanced.

In addition to providing new insights into normal neurofilament trafficking in the neuron, these findings might have considerable clinical importance. Abnormal aggregates of neurofilaments in motor neurons have long been recognized as characteristics of the neurodegenerative disease amyotrophic lateral sclerosis (ALS), and defective neurofilament trafficking has been implicated in the mechanism that leads to neuronal death in this condition. Intriguingly, increased $\mathrm{Cdk} 5$ activity has also been observed in ALS. The findings of Shea $e t$ al. provide a possible mechanistic link between these phenomena. Their work also raises some interesting issues from the perspective of basic neuronal biology. For example, if the normal trafficking of neurofilaments is regulated by Cdk5mediated phosphorylation, how does the neuron partition the activity of the enzyme so that neurofilament subunits are only able to aggregate once they reach the axon?

Heather Wood

\section{(D) References and links}

ORIGINAL RESEARCH PAPER Shea, T. B. et al. Cdk5

regulates axonal transport and phosphorylation of

neurofilaments in cultured neurons. J. Cell Sci. 117, 933-941 (2004)

FURTHER READING Cleveland, D. W. \& Rothstein, J. D. From Charcot to Lou Gehrig: deciphering selective motor neuron death in ALS. Nature Rev. Neurosci. 2, 806-819 (2001)

WEB SITES

Encyclopedia of Life Sciences: http://www.els.net/ amyotrophic lateral sclerosis | cell cycle: regulation by cyclins

\title{
Driving whisker movement
}

The motor cortex consists of millions of neurons that are active in complex spatial and temporal patterns to generate behaviour. But what contribution, if any, do action potentials in individual neurons make to movement? Brecht
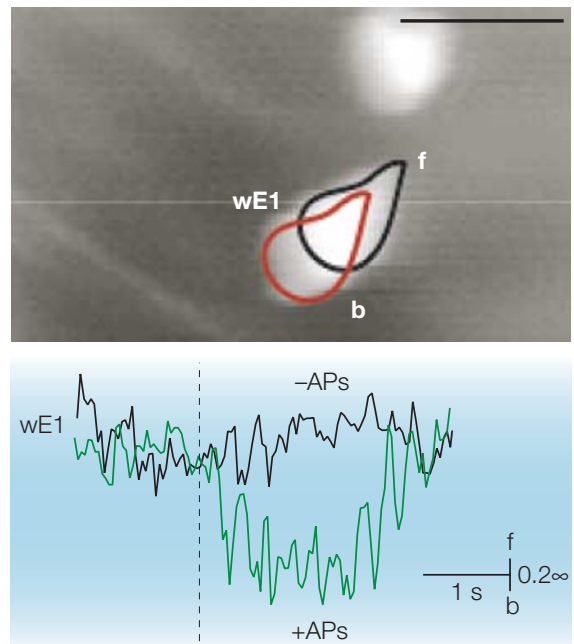

Upper panel shows the forward (f) and backward (b) position of whisker E1 ( $w E 1$ ) during stimulation of a single layer-6 pyramidal cell. Scale bar, $10 \mathrm{~mm}$. Lower panel is a plot of the position of whisker $\mathrm{E} 1$ over time during control and stimulation trials. The black trace corresponds to the control trial, in which subthreshold or hyperpolarizing pulses were used. Dashed line shows the onse of the action potential (AP) train. Image courtesy of T. Margrie, Department of Physiology, University of London. and colleagues addressed this question directly by making intracellular recordings from vibrissae motor cortex pyramidal neurons in anaesthetized rats. In technically challenging experiments, the authors injected small amounts of current into the recorded neurons while simultaneously monitoring the position of individual whiskers.

In about a quarter of the neurons, trains of action potentials - triggered by the intracellular current injection - were associated with small back and forth motions in local groups of whiskers. The latency for whisker deflection was quite long, and movements often outlasted the stimulation considerably. This indicates that the action potentials that were triggered in the recorded neuron influenced a downstream circuit that was then responsible for pattern generation. This influence varied with action potential frequency, and, interestingly, with the laminar location of the recorded neuron. For example, the phase of the whisking movements relative to the action potential stimulus train was constant in the case of layer 5 neurons, but not layer 6 neurons.

Activity in the motor cortex has previously been correlated with behaviour using electrophysiological recordings. Furthermore, extracellular microstimulation techniques in behaving animals have enabled researchers to perturb ongoing activity to test theories about cortical representations of movement. The intracellular stimulation technique used by Brecht and colleagues has the advantage of exquisite control over membrane potential and action potential generation coupled with high spatial resolution - the currents used are so low that there is little danger of stimulating fibres of passage.

However, the trade-off is that animals have to be anesthetized to maintain stable recordings, and the effects of anaesthesia are likely to be significant. In fact, the authors provide some preliminary evidence that bursts of action potentials in single neurons are even more effective in awake animals: in a few cases, they were able to maintain a stable intracellular recording while they antagonized the anaesthesia pharmacologically. As the animals were waking up, the whisker responses to intracellular stimulation were larger and faster than in any of the cases where the animals were more deeply anaesthetized.

So, is it appropriate to conclude that every spike conveys a message? The results are provocative, but more work needs to be done to determine how well the observations generalize to awake animals and to more complex motor systems.

John Spiro, Senior Editor, Nature

\section{(2) References and links}

ORIGINAL RESEARCH PAPER Brecht, M. et al. Whisker

movements evoked by stimulation of single pyramidal cells in rat motor cortex. Nature 427, 704-710 (2004) 